\title{
The New Approach to the Detection of the Abrupt Change of Fast Fluctuating Random Processes in the Conditions of Parametric Prior Uncertainty
}

\author{
Oleg V. Chernoyarov \\ Dept. of Radio engineering Devices \\ National Research University "MPEI” (MPEI) \\ Moscow, Russia \\ o_v_ch@mail.ru \\ Boris I. Shakhtarin \\ Dept. of Self-contained Information and Control Systems \\ Bauman Moscow State University (BMSU) \\ Moscow, Russia \\ shakhtarin@mail.ru
}

\author{
Alexander P. Ermakov, Dmitry K. Proskurin \\ Dept. of Information Technologies and Computer-aided \\ Design in Building \\ Voronezh State University of Architecture and Civil Engi- \\ neering (VSUACE) \\ Voronezh, Russia \\ pdk@vgasu.vrn.ru
}

\begin{abstract}
In this study work we introduce a technically simple way of the detection of an abrupt change in parameters of fast fluctuating Gaussian processes in the conditions of parametric prior uncertainty. For this purpose, we suggest new approximations of solving statistics under various hypotheses. As an example of the mathematical expectation jumping of a random process, the appropriate detection algorithm is synthesized and the method of finding of the analytical expressions for the characteristics of its operating effectiveness is illustrated. Applying statistical computer modeling, we have found that the proposed method of the detection of the abrupt changes in parameters of fastfluctuating random processes is operable, and the theoretical formulas for the detection characteristics well conform with the corresponding experimental data in a wide range of parameter values of the analyzed process.
\end{abstract}

Keywords-random process; abrupt change; detection; maximum likelihood method; parametrical prior uncertainty; local Markov approximation method; statistical computer modeling.

\section{INTRODUCTION}

The problem of detection of the abrupt change at some time point in the characteristics of the analyzed random process has a wide application in communications, radio- and hydrolocations, remote signaling and control systems etc. [1-3, etc.]. In the known literature the statement of this problem is accompanied by the assumption that the observable data realization has a normal distribution, and also, as a rule, the additional restrictions are imposed referring to the processed samples being uncorrelated [1], to the mathematical model classes of the information signal [2,3], and etc.

In the present work we suggest a new approach for the detection of the abrupt change of the fast fluctuating random processes in the conditions of parametric prior uncertainty and taking as an example a definition of the mathematical expecta-

The reported study was supported by Russian Foundation for Basic Research (research projects No. 13-08-00735, 13-08-97538) and Russian Science Foundation (research project No. 14-29-00208) tion jumping of the Gaussian random process with unknown intensity. Analytically such process can be presented as follows

$$
\xi(t)=v(t)+a_{01}+\left(a_{02}-a_{01}\right) \theta\left(t-\lambda_{0}\right),
$$

where $\theta(t)=0$ if $t<0$ and $\theta(t)=1$ if $t \geq 0-$ Heaviside function, $\lambda_{0}-$ the moment of possible abrupt change, $a_{01}, a_{02}-$ mean values (mathematical expectations) of the process $\xi(t)$ under $t<\lambda_{0}$ and $t \geq \lambda_{0}$ accordingly, and $v(t)$ - stationary centered Gaussian random process. We will present spectral density of the process $v(t)$ as $[4,5]$

$$
G(\omega)= \begin{cases}d_{0} / 2, & |\omega| \leq \Omega / 2 \\ 0, & |\omega|>\Omega / 2\end{cases}
$$

Here $\Omega$ - bandwidth, $d_{0}$ - intensity of the process $v(t)$ (or $\xi(t)$ ), defining its dispersion $D_{0}=d_{0} \Omega / 4 \pi$ also, and as the process $\xi(t)$ is fast fluctuating (on a problem statement), we will consider that the following condition is satisfied:

$$
\mu_{\text {min }}=T_{\text {min }} \Omega / 4 \pi>>1
$$

where $T_{\min }=\min \left(\lambda_{0}, T-\lambda_{0}\right)$.

We believe that process (1) is observed against additive Gaussian white noise $n(t)$ with one-sided spectral density $N_{0}$. As a result the mix

$$
x(t)=\xi(t)+n(t), \quad t \in[0, T]
$$


is accessible to be observed. With the observable realization (4) it is necessary to make a decision on a presence or an absence of the constant component abrupt change in the observable data. In general, the parameters $a_{01}, a_{02}, d_{0}$ are unknown.

\section{SYNTHESIS OF THE DETECTION ALGORITHM OF THE ABRUPT CHANGE IN MATHEMATICAL EXPECTATION}

For the solution of the detection problem of abrupt change in mathematical expectation of the process $\xi(t)$, we separate two possible cases: 1) $a_{01}=a_{02}$, i.e. abrupt change is absent ( $H_{0}$ hypothesis); 2) $a_{01} \neq a_{02}$ ( $H_{1}$ hypothesis). We will solve the problem of the specified hypothesis testing by the use of the maximum likelihood method. For this purpose we will apply the approach connected with introduction of auxiliary hypothesis $H: x(t)=n(t)$ and will designate $L_{0}\left(a_{1}, d\right), L_{1}\left(\lambda, a_{1}, a_{2}, d\right)$ as logarithms of the functionals of likelihood ratio for hypotheses $H_{0}, H_{1}$ against alternative $\mathrm{H}$ accordingly. Further, following [4, 5], and neglecting sizes of order of process $\xi(t)$ time correlation (under satisfying Eq. (3)), the next essentially simplified (in comparison with the known) approximations for solving statistics $L_{0}\left(a_{1}, d\right), L_{1}\left(\lambda, a_{1}, a_{2}, d\right)$ can be received:

$$
\begin{gathered}
L_{0}\left(a_{1}, d\right)=\frac{d}{N_{0}\left(N_{0}+d\right)} \int_{0}^{T} y^{2}(t) d t+ \\
+\frac{2 a_{1}}{N_{0}+d} \int_{0}^{T} x(t) d t-\frac{a_{1}^{2} T}{N_{0}+d}-\frac{\Omega T}{4 \pi} \ln \left(1+\frac{d}{N_{0}}\right) \\
H_{1}: \quad \\
+\frac{2 a_{1}}{L_{0}+d} \int_{0}^{\lambda} x(t) d t-\frac{a_{1}^{2} \lambda}{N_{0}+d}+ \\
+\frac{\left.2 a_{2}, a_{2}, d\right)=\frac{d}{N_{0}\left(N_{0}+d\right)} \int_{0}^{T} y^{2}(t) d t+}{N_{0}+d} \int_{\lambda}^{T} x(t) d t-\frac{a_{2}^{2}(T-\lambda)}{N_{0}+d}-\frac{\Omega T}{4 \pi} \ln \left(1+\frac{d_{1}}{N_{0}}\right) .
\end{gathered}
$$

In Eq. (5) it is designate: $y(t)=\int_{-\infty}^{\infty} x\left(t^{\prime}\right) h\left(t-t^{\prime}\right) d t^{\prime}$ is the output signal of the filter with the transfer function $H(\omega)$, satisfying to a condition $|H(\omega)|^{2}=2 G(\omega) / d_{0}(2)$, and $\lambda, a_{1}, a_{2}, d$ are current values of parameters $\lambda_{0}, a_{01}, a_{02}, d_{0}$, accordingly. The choice is made in favor of $H_{1}$ hypothesis, if $[4,6]$

$$
\max _{\lambda \in\left[\Lambda_{1}, \Lambda_{2}\right], a_{1}, a_{2}, d} L_{1}\left(\lambda, a_{1}, a_{2}, d\right)-\max _{a_{1}, d} L_{0}(a, d)>c .
$$

In the opposite case, the decision is made in favor of $H_{0}$ hypothesis. Here $\left[\Lambda_{1}, \Lambda_{2}\right]$ is a prior interval of possible values of $\lambda_{0}$ parameter and $c$ is threshold defined by set optimality criterion [6].

Under unknown parameters $\lambda_{0}, a_{01}, a_{02}, d_{0}$ maximization of functionals (5) on variables $a_{1}, a_{2}, d$ can be performed analytically. As a result we find

$$
\begin{gathered}
L_{0 \max }=\max _{a_{1}, d} L_{0}\left(a_{1}, d\right)=\frac{\Omega T}{4 \pi}\left\{\frac{4 \pi}{N_{0} \Omega T} \int_{0}^{T} y^{2}(t) d t-\right. \\
\left.-\ln \left[\frac{4 \pi}{N_{0} \Omega T}\left(\int_{0}^{T} y^{2}(t) d t-\frac{1}{T}\left(\int_{0}^{T} x(t) d t\right)^{2}\right)\right]-1\right\},
\end{gathered}
$$

$$
\begin{gathered}
L_{1 \text { max }}(\lambda)=\max _{a_{1}, a_{2}, d} L_{1}\left(\lambda, a_{1}, a_{2}, d\right)=L_{0 \text { max }}+ \\
+\frac{\Omega T}{4 \pi} \ln \left[\frac{\int_{0}^{T} y^{2}(t) d t-\frac{1}{T}\left(\int_{0}^{T} x(t) d t\right)^{2}}{\int_{0}^{T} y^{2}(t) d t-\frac{1}{\lambda}\left(\int_{0}^{\lambda} x(t) d t\right)^{2}-\frac{1}{T-\lambda}\left(\int_{\lambda}^{T} x(t) d t\right)^{2}}\right]
\end{gathered}
$$

Then, from Eqs. (6), (7) follows that maximum likelihood detection algorithm of abrupt change in mathematical expectation of Gaussian process with unknown intensity has the appearance

$\max _{\lambda \in\left[\Lambda_{1}, \Lambda_{2}\right]} M(\lambda)_{<}^{>} c, M(\lambda)=M_{1}^{2}(\lambda) / \lambda+M_{2}^{2}(\lambda) /(T-\lambda)-M_{3}^{2} / T$

and also, is invariant to spectral density of white noise. In Eq. (8) it is designate:

$$
M_{1}(\lambda)=\int_{0}^{\lambda} x(t) d t, \quad M_{2}(\lambda)=\int_{\lambda}^{T} x(t) d t, \quad M_{3}=\int_{0}^{T} x(t) d t
$$

We will characterize detection quality with using type I (false alarm) $\alpha$ and II (missing) $\beta$ error probabilities $[4,6]$. For a finding $\alpha$ and $\beta$ we put into consideration the normalized functionals

$$
\tilde{M}_{i}(l)=M_{i}(\lambda) \sqrt{2 / T\left(N_{0}+d_{0}\right)}, \quad \tilde{M}_{3}=M_{3} \sqrt{2 / T\left(N_{0}+d_{0}\right)}
$$

$i=1,2$, and present them as the sums of signal and noise functions [6]: 


$$
\tilde{M}_{i}(l)=S_{i}(l)+N_{i}(l), \quad \tilde{M}_{3}=S_{3}+N_{3} .
$$

Here $\quad S_{i}(l)=\left\langle\tilde{M}_{i}(l)\right\rangle \quad, \quad S_{3}=\left\langle\tilde{M}_{3}\right\rangle \quad$ are $\quad$ signal, $N_{i}(l)=\tilde{M}_{i}(l)-\left\langle\tilde{M}_{i}(l)\right\rangle, N_{3}=\tilde{M}_{3}-\left\langle\tilde{M}_{3}\right\rangle$ are noise functions, $l=\lambda / T$ is current value of the normalized parameter $l_{0}=\lambda_{0} / T$, and the averaging $\langle\cdot\rangle$ is implemented through all possible realizations $x(t)$ with fixed values of all unknown parameters $\lambda_{0}, a_{01}, a_{02}, d_{0}$. While executing the ratio (3), for signal functions and correlation functions of noise functions specified above we have

$$
\begin{gathered}
S_{1}(l)=z_{01} l+\Delta z \max \left(0, l-l_{0}\right), \quad S_{3}=z_{01}+\Delta z\left(1-l_{0}\right), \\
S_{2}(l)=z_{01}(1-l)+\Delta z\left[1-\max \left(l, l_{0}\right)\right], \\
\left\langle N_{1}\left(l_{1}\right) N_{1}\left(l_{2}\right)\right\rangle=\min \left(l_{1}, l_{2}\right), \quad\left\langle N_{3}^{2}\right\rangle=1, \\
\left\langle N_{2}\left(l_{1}\right) N_{2}\left(l_{2}\right)\right\rangle=1-\max \left(l_{1}, l_{2}\right),
\end{gathered}
$$

where $\Delta z=z_{02}-z_{01}$ and $z_{0 i}=a_{0 i} \sqrt{2 T /\left(N_{0}+d_{0}\right)}, i=1,2$ are signal-to-noise ratios (SNR) for constant components $a_{0 i}$ on all observation interval.

At first let us put that abrupt change in mathematical expectation of the process $\xi(t)(1)$ is absent, i.e.

$$
z_{01}=z_{02}
$$

Then false-alarm probability $\alpha$ can be written down in a kind

$$
\alpha=P\left[\max _{l \in\left[\tilde{\Lambda}_{1}, \tilde{\Lambda}_{2}\right]} \tilde{M}(l)>u\right]=1-P_{N}(u),
$$

where $\quad \tilde{M}(l)=2 M(\lambda) / T\left(N_{0}+d_{0}\right) \quad, \quad \tilde{\Lambda}_{1,2}=\Lambda_{1,2} / T \quad$, $u=2 c / T\left(N_{0}+d_{0}\right)$ is a normalized threshold,

$$
P_{N}(u)=P[\tilde{M}(l)<u], \quad l \in\left[\tilde{\Lambda}_{1}, \tilde{\Lambda}_{2}\right] .
$$

Taking into account Eqs. (10)-(12) we present the functional $\tilde{\mathrm{M}}(l)$ as

$$
\tilde{M}(l)=\left[N_{1}(l) \sqrt{(1-l) / l}-N_{2}(l) \sqrt{l /(1-l)}\right]^{2} .
$$

Further, in Eq. (15) let us execute the change of variables:

$$
\theta=\ln [l /(1-l)], \theta \in\left[\Theta_{1}, \Theta_{2}\right], \Theta_{i}=\ln \left[\tilde{\Lambda}_{i} /\left(1-\tilde{\Lambda}_{i}\right)\right], i=1,2
$$

Then, the probability (14) can be overwritten as

$$
P_{N}(u)=P\left[X^{2}(\theta)<u\right], \quad \theta \in\left[\Theta_{1}, \Theta_{2}\right],
$$

where $X(\theta)$ is Gaussian random process with zero mathematical expectation and correlation function $\left\langle X\left(\theta_{1}\right) X\left(\theta_{2}\right)\right\rangle=\exp \left(-\left|\theta_{2}-\theta_{1}\right| / 2\right)$. Consequently, on the basis of results [7] for probability (14) we find

$$
P_{N}(u)= \begin{cases}\exp \left[-\left(\Theta_{2}-\Theta_{1}\right) \sqrt{u / 2 \pi} \exp (-u / 2)\right], & u \geq 1, \\ 0, & u<1 .\end{cases}
$$

Accordingly, the expression for false-alarm probability (13) goes over

$$
\alpha= \begin{cases}1-\exp \left[-\left(\Theta_{2}-\Theta_{1}\right) \sqrt{u / 2 \pi} \exp (-u / 2)\right], & u \geq 1, \\ 1, & u<1 .\end{cases}
$$

Under $\tilde{\Lambda}_{1} \rightarrow 0, \tilde{\Lambda}_{2} \rightarrow 1$, as appears from Eq. (16), we have $\Theta_{1} \rightarrow-\infty, \Theta_{2} \rightarrow \infty$. However, according to the conducted empirical studies the difference $\Theta_{2}-\Theta_{1}$ in Eq. (17) can be accepted finite and equal

$$
\Theta_{2}-\Theta_{1}=12
$$

Accuracy of the formula (17) increases with $u$ and ratio $\tilde{\Lambda}_{2}\left(1-\tilde{\Lambda}_{1}\right) / \tilde{\Lambda}_{1}\left(1-\tilde{\Lambda}_{2}\right)$.

Let us put now that $z_{01} \neq z_{02}$. In this case the abrupt change missing probability is

$$
\beta=P\left[\max _{l \in\left[\tilde{\Lambda}_{1}, \tilde{A}_{2}\right]} \tilde{M}(l)<u\right]=P\left[\underset{l \in\left[\tilde{A}_{1}, \tilde{L}_{2}\right]}{\tilde{M}(l)}<u\right]
$$

Let us consider that $|\Delta z|>>1$. Then taking into account Eq. (11) the signal function $S(l)=\langle\tilde{M}(l)\rangle$ and correlation function $\left\langle N\left(l_{1}\right) N\left(l_{2}\right)\right\rangle$ of noise function $N(l)=\tilde{M}(l)-\langle\tilde{M}(l)\rangle$ of the normalized functional $\tilde{M}(l)(8)$ allow approximations

$$
S(l)=\Delta z^{2} \begin{cases}\left(1-l_{0}\right)^{2} y, & l \leq l_{0}, \\ l_{0}^{2} / y, & l>l_{0},\end{cases}
$$

$$
\left\langle N\left(l_{1}\right) N\left(l_{2}\right)\right\rangle=4 \Delta z^{2} \begin{cases}\left(1-l_{0}\right)^{2} \min \left(y_{1}, y_{2}\right), & l_{1}, l_{2} \leq l_{0}, \\ l_{0}^{2} \min \left(1 / y_{1}, 1 / y_{2}\right), & l_{1}, l_{2}>l_{0},\end{cases}
$$


where $y=l /(1-l), y_{i}=l_{i} /\left(1-l_{i}\right), i=1,2$.

From Eq. (20) follows that on intervals $\left[\tilde{\Lambda}_{1}, l_{0}\right]$ and $\left(l_{0}, \tilde{\Lambda}_{2}\right]$ the functional $\tilde{M}(l)$ is Gaussian Markovian diffusion process with drift $K_{1}$ and diffusion $K_{2}$ coefficients of a kind

$$
K_{1}=\Delta z^{2}\left\{\begin{array}{l}
\left(1-l_{0}\right)^{2}, l \leq l_{0}, \\
-l_{0}^{2} / y^{2}, l>l_{0},
\end{array} K_{2}=4 \Delta z^{2}\left\{\begin{array}{cc}
\left(1-l_{0}\right)^{2}, & l \leq l_{0}, \\
l_{0}^{2} / y^{2}, & l>l_{0} .
\end{array}\right.\right.
$$

From here, using a technique presented in [8], for probability (19) we find

$$
\begin{gathered}
\beta=\frac{\exp \left[-\frac{a^{2} \tilde{y}_{1}^{2}}{2\left(b \tilde{y}_{1}+\sigma_{1}^{2}\right)}\right] \int_{0}^{\infty} \exp \left[\frac{a \tilde{y}_{1}(\tilde{u}-x)}{b \tilde{y}_{1}+\sigma_{1}^{2}}\right] \times}{\sqrt{2 \pi\left(b \tilde{y}_{1}+\sigma_{1}^{2}\right)}} \times\left\{\operatorname { e x p } [ - \frac { ( \tilde { u } - x ) ^ { 2 } } { 2 ( b \tilde { y } _ { 1 } + \sigma _ { 1 } ^ { 2 } ) } ] \Phi \left[\frac{\tilde{y}_{1}\left(a \sigma_{1}^{2}+b \tilde{u}\right)+\sigma_{1}^{2} x}{\left.\sigma_{1} \sqrt{b \tilde{y}_{1}\left(b \tilde{y}_{1}+\sigma_{1}^{2}\right)}\right]-}\right.\right. \\
-\exp \left[-\frac{4 a \sigma_{1}^{2} x+b(\tilde{u}-x)^{2}}{2 b\left(b \tilde{y}_{1}+\sigma_{1}^{2}\right)}\right] \Phi\left[\frac{\tilde{y}_{1}\left(a \sigma_{1}^{2}+b \tilde{u}\right)-\sigma_{1}^{2} x}{\left.\left.\sigma_{1} \sqrt{b \tilde{y}_{1}\left(b \tilde{y}_{1}+\sigma_{1}^{2}\right)}\right]\right\} \times}\right. \\
\times\left\{\Phi\left(a \sqrt{\frac{\tilde{y}_{2}}{b}}+\frac{x}{\sqrt{b \tilde{y}_{2}}}\right)-\exp \left(-\frac{2 a x}{b}\right) \Phi\left(a \sqrt{\left.\frac{\tilde{y}_{2}}{b}-\frac{x}{\sqrt{b \tilde{y}_{2}}}\right)}\right)\right\} d x .
\end{gathered}
$$

Here it is designated: $\tilde{y}_{1}=y_{0}-Y_{1}, \tilde{y}_{2}=Y_{2}-y_{0}, \tilde{u}=u-S_{1}$, $Y_{i}=\tilde{\Lambda}_{i} /\left(1-\tilde{\Lambda}_{i}\right), i=1,2, S_{1}=\tilde{S}\left(\tilde{\Lambda}_{1}\right)=\Delta z^{2}\left(1-l_{0}\right)^{2} \tilde{\Lambda}_{1} /\left(1-\tilde{\Lambda}_{1}\right)$, $a=\left|K_{1}\right|_{l=l_{0}}=\Delta z^{2}\left(1-l_{0}\right)^{2} \quad, \quad b=\left.K_{2}\right|_{l=l_{0}}=4 \Delta z^{2}\left(1-l_{0}\right)^{2}$, $y_{0}=l_{0} /\left(1-l_{0}\right), \sigma_{1}^{2}=\left\langle\tilde{N}^{2}\left(\tilde{\Lambda}_{1}\right)\right\rangle=4 \Delta z^{2}\left(1-l_{0}\right)^{2} \tilde{\Lambda}_{1} /\left(1-\tilde{\Lambda}_{1}\right)$.

Under $\tilde{\Lambda}_{1} \rightarrow 0, \tilde{\Lambda}_{2} \rightarrow 1\left(Y_{1} \rightarrow 0, Y_{2} \rightarrow \infty\right)$ the expression (21) becomes simpler essentially and takes the form

$$
\begin{gathered}
\beta=\Phi\left(-\frac{a y_{0}-u}{\sqrt{b y_{0}}}\right)-\exp \left(\frac{2 a\left(2 a y_{0}-u\right)}{b}\right) \Phi\left(-\frac{3 a y_{0}-u}{\sqrt{b y_{0}}}\right)- \\
-\exp \left(\frac{2 a u}{b}\right) \Phi\left(-\frac{a y_{0}+u}{\sqrt{b y_{0}}}\right)+\exp \left(\frac{4 a\left(a y_{0}+u\right)}{b}\right) \Phi\left(-\frac{3 a y_{0}+u}{\sqrt{b y_{0}}}\right)
\end{gathered}
$$

Accuracy of the formulas (21), (22) increases with $\Delta z$ and $u$.

\section{RESULTS OF STATISTICAL MODELING}

In order to establish the applicability range of asymptotically exact formulas for detection characteristics the statistical computer modeling of the algorithm (8) operation was carried out. During the modeling the samples of realizations of functionals $\tilde{M}_{i}(l), i=1,2, \tilde{M}_{3}(9)$ were formed within the interval $(0,1)$ with digitization step $\Delta=10^{-4}$ on both normalized time $\tilde{t}=t / T$ and current value $l$ of the parameter $l_{0}$ in abrupt change presence or absence. Further, for each realization $\tilde{M}(l)$ (8) the comparison of its greatest sample with threshold $u$ was executed and false-alarm and missing probabilities were found.

In Figs. 1, 2 some results of statistical modeling are presented where corresponding theoretical dependences are shown also. Each experimental value was received as a result of processing of not less than $10^{5}$ realizations $\tilde{M}(l)$ under $\mu=T \Omega / 4 \pi=200$. In Fig. 1 the theoretical dependence of false-alarm probability $\alpha(u)(17)$, (18) is traced by solid line, and in Fig. 2 the theoretical dependences of missing probability $\beta(\Delta z)$ (22) are plotted for $l_{0}=0.05$ (solid line), 0.125 (dashed line), 0.5 (dash-and-dot line). Experimental values of detection characteristics are designated by squares (Fig. 1) and by squares, crosses, circlets for $l_{0}=0.05,0.125,0.5$ (Fig. 2). The threshold size $u$ was defined from Eq. (17) by Neumann-Pirson criterion, according to the specified level of false-alarm probability $\alpha=0.01$, so $u=15.043$.

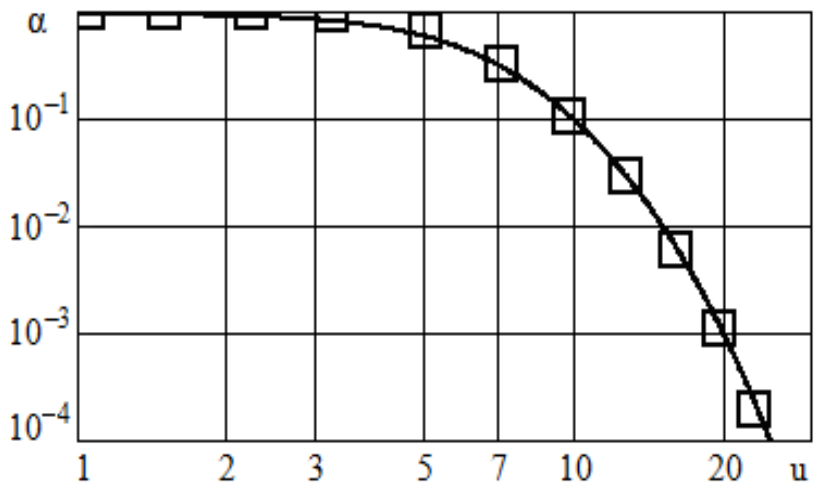

Fig. 1. False-alarm probability.

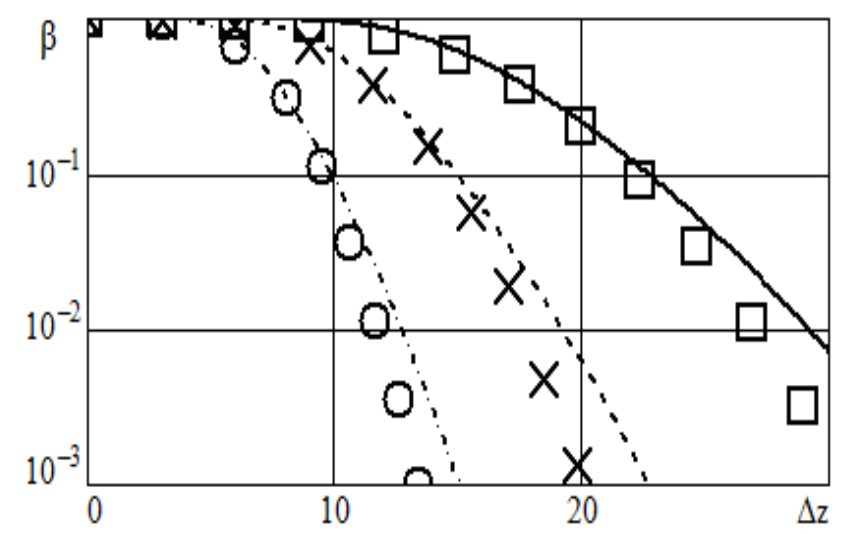

Fig. 2. Missing probability. 
As follows from Figs. 1, 2, theoretical dependences for probabilities $\alpha$ (17) and $\beta$ (22) well approximate experimental data in a wide range of values of analyzed process $\xi(t)$ (1) parameters. During background studies it was established that the algorithm (8) can be used for abrupt change detection of mathematical expectation of many widespread non-Gaussian random processes without appreciable sacrifice of accuracy.

\section{CONCLUSION}

In order to detect the change-point time of abruptly changing fast fluctuating Gaussian processes in the conditions of parametric prior uncertainty, the maximum likelihood method can be effectively used. Application of this method allows us, while neglecting the sizes of order of the analyzed random process correlation time, to receive detection algorithms of abrupt changing random processes, technically more simple essentially in comparison with the known analogues. With the help of a local Markov approximation method, the closed analytical expressions for the performance indices of the given algorithms can be found. By means of the statistical computer modeling, we established that the obtained theoretical results well coincide with corresponding experimental data in a wide range of parameter values of the observable process. Additional researches show that detectors synthesized on the basis of the proposed approach can be also used for the analysis of nonGaussian abrupt changing random processes without appreciable loss in functioning.

\section{REFERENCES}

[1] A.A. Zhiglyavsky, A.E. Kraskovsky, Detection of Abrupt Changes in Random Processes for Radio engineering Tasks [in Russian], Leningrad State University, Leningrad, 1988.

[2] M. Basseville, I.V. Nikiforov, Detection of Abrupt Changes: Theory and Application, Prentice-Hall, Englewood Cliffs, NJ, 1993.

[3] E.-S. Akhouayri, E.H.A. Laasri, D. Agliz, A. Atmani "Signal Stationary Testing and Detecting of its Abrupt Change", Electronics, Communications and Photonics Conference (SIECPC), 2011 Saudi International: Riyadh Saudi Arabia, 2011, pp. 1-5.

[4] A.P. Trifonov, E.P. Nechaev, V.I. Parfenov, Detection of Stochastic Signals with Unknown Parameters [in Russian], Voronezh State University, Voronezh, 1991.

[5] O.V. Chernoyarov, "The Efficiency of Reception of a Random Pulse Signal with Unknown Parameters under the Conditions of Detuned Duration", Telecommunications and Radio Engineering, vol. 72, No. 1, 2013, pp. 1-23.

[6] A.P. Trifonov, Yu.S. Shinakov, Joint Discrimination of Signals and Estimation of Their Parameters Against Background [in Russian], Moscow, Radio i Svyaz', 1986.

[7] O.V. Chernoyarov, A.V. Salnikova, Ya.A. Kupriyanova, "Definition of probability characteristics of the absolute maximum of non-Gaussian random processes by example of Hoyt process", American Journal of Theoretical and Applied Statistics, vol. 2, No. 3, 2013, pp. 54-60.

[8] A.P. Trifonov, Y.E. Korchagin, P.A. Kondratovich, "Efficiency of estimating duration of a signal with unknown amplitude", Radioelectronics and Communications Systems, vol. 54, No. 11, 2011, pp. 581-591. 